\title{
ACAÇA ESPORTIVA E OS EFEITOS DO CHUMBO NAS AVES DO SUL DO BRASIL
}

\author{
Ricardo $\operatorname{Lau}^{1}$ \\ ${ }^{1}$ Universidade do Vale do Rio dos Sinos (UNISINOS), Centro de Ciências da Saúde, Programa de Pós-Graduação em Biologia. Avenida \\ Unisinos, 950, Bairro Cristo Rei, São Leopoldo, RS, Brasil. CEP: 93022-750 \\ E-mail: biokdee@hotmail.com
}

\section{RESUMO}

O chumbo $(\mathrm{Pb})$ é um metal reconhecidamente tóxico aos organismos quando presente por muito tempo e em altos níveis no ambiente. A contaminação, verificada através de dados clínicos e laboratoriais, resulta em efeitos fisiológicos adversos e graves e, em casos extremos, pode levar ao óbito. As principais fontes de acúmulo de $\mathrm{Pb}$ localizam-se em áreas intensamente urbanizadas devido a atividades automotivas e industriais. O metal é utilizado como munição de caça principalmente em países em desenvolvimento e é fonte conhecida de contaminação de aves e ambientes. O objetivo é revisar fontes de informação sobre caça esportiva com a presença da munição a base de Pb desde o Século XIX. Adicionalmente, analiso a questão da caça esportiva no sul do Brasil, em uma das áreas mais importantes da atividade cinegética (arte de caçar, especialmente com auxilio de cães), no estado do Rio Grande do Sul. Verifiquei que os estudos de contaminação por Pb em aves realizados até o momento são focados principalmente em aves aquáticas, sendo que para aves estritamente terrestres há poucos registros. Grande parte de estudos de contaminação por munição de caça com Pb foram realizados em países desenvolvidos como nos EUA, Canadá, Reino Unido e Espanha, onde a caça esportiva com munição contendo Pb possui mais de 100 anos de história. Nestes países, no entanto, a munição de Pb já foi banida ou substituída por outros materiais. No sul do Brasil, as pesquisas sobre a problemática ainda são escassas, evidenciando a importância de estudos mais aprofundados para obterem-se dados concretos e substanciais necessários em tomada de decisões.

Palavras-chave: aves campestres; chumbo; cinegética; contaminação; Rio Grande do Sul.

\section{ABSTRACT -SPORT HUNTING AND THE LEAD EFFECT ON BIRDS FROM SOUTHERN BRAZIL}

Lead $(\mathrm{Pb})$ is recognized as a toxic metal to organisms when present for a long time and also when it is found in high levels in the environment. The contamination, verified through clinical and laboratorial data, results in adverse and severe physiological effects and, in extreme cases, death. The main build-up sources of $\mathrm{Pb}$ are located in highly urban areas due to automotive and industrial activities. The metal is used as hunting ammunition, mainly in developing countries and it is a known contamination source for birds and environments. The objective is to review information sources on sport hunting with the use of $\mathrm{Pb}$ based ammunition since the nineteenth century. I analyze the matter of sport hunting in the State of Rio Grande do Sul, which is one of the most important areas of hunting activity in Brazil. I verified that the studies on $\mathrm{Pb}$ contamination on birds so far are mainly focused on waterbirds and that there have been few records on terrestrial birds. The majority of studies focusing on $\mathrm{Pb}$ contamination through its use in ammunition have been done on developed countries such as USA, Canada, United Kingdom and Spain, where sport hunting with $\mathrm{Pb}$ ammunition has over 100 years of use. In those countries, $\mathrm{Pb}$ ammunition has been banned or replaced by other materials. In southern Brazil, researches on this issue are scarce, which reinforces the need of detailed studies in order to obtain more precise and substantial data necessary on decision-taking.

Keywords: contamination; lead; Rio Grande do Sul; sport hunting; terrestrial birds.

\section{INTRODUÇÃO}

O chumbo $(\mathrm{Pb})$ é um metal pesado, com baixa temperatura de fusão, cor cinza-azulado metalizado e encontrado naturalmente na crosta terrestre (USDHHS 2007). É um elemento que em níveis altos e/ou depositado por muito tempo no meio ambiente pode ser tóxico e fatal aos seres vivos (Davidson \& Nettles
1997, Friend et al. 1999, Beyer et al. 2004, US-DHHS 2007, Rattner 2009). As inserções no meio urbano provem principalmente de atividades automotivas, como o uso e reciclagem de baterias e aditivos de combustíveis, bem como de indústrias de pigmentos e efluentes industriais diversos. Na área rural, a mineração de carvão, o uso de fertilizantes fosfatados e alguns adubos orgânicos, como esterco de animais, 
lixo domiciliar e biossólidos, são as principais fontes (Flores \& Martins 1997, Tsutiya 1999, Beyer et al. 2004, US-DHHS 2007).

Durante muito tempo, a atividade cinegética (arte de caçar, especialmente com auxilio de cães) foi uma das fontes adicionais de deposição de $\mathrm{Pb}$ no ambiente, sobretudo em países com muitos praticantes de caça, como E.U.A., Canadá, Japão, Reino Unido e Espanha DL Guadagnin \& LFC Perello (dados não publicados). Recentemente estes países baniram a utilização do $\mathrm{Pb}$ em munição de caça e/ou sugeriram a troca por outros elementos não tóxicos (Tranel \& Kimmel 2008, Pain et al. 2009, Scheuhammer 2009, US-FWS 2014). Em países onde a caça é tradicional e permitida, a munição a base de $\mathrm{Pb}$ foi proibida para áreas úmidas (E.U.A., 1991; Inglaterra, 1999; País de Gales, 2002; Escócia, 2004; Irlanda do Norte, 2009), e indicada a troca por munição alternativa composta por matérias-primas não-toxicas, como o bismuto, ferro, aço, tungstênio, cobre, níquel, bronze (US-FWS 2014, Beintema 2003), já testadas. No Brasil são escassos estudos sobre o tema, sendo o estado do Rio Grande do Sul onde se relatam os poucos trabalhos, pois manteve a caça regulamentada da década de 70 até o ano de 2005. Nesta região, a caça ocorria em extensos campos da metade sul do território e áreas úmidas (banhados, lagoas e lavouras de arroz após colheita). Juntamente com a caça legalizada, o estado manteve levantamentos, pesquisas e monitoramentos sistemáticos sobre a dinâmica das populações de aves que podiam ser caçadas (Duarte 2005, Duarte et al. 2007).

Costa (2006) apontou que poderia haver contaminação por $\mathrm{Pb}$ (saturnismo) principalmente em aves aquáticas no estado, porém não apresentou dados a respeito. Análises do risco de contaminação por este metal no Rio Grande do Sul foram sugeridas por Guadagnin et al. (2007) e DL Guadagnin \& LFC Perello (dados não publicados). Eles sugerem a realização de estudos para aprofundar o conhecimento sobre o tema e gerar informações para a gestão. Assim, entre 2009 e 2011 foram realizadas pesquisas de campo e laboratoriais para analisar os eventuais níveis de contaminação por $\mathrm{Pb}$ decorrente da munição de caça em setores campestres do Rio Grande do Sul (Lau 2011).
O objetivo deste trabalho é realizar uma revisão sobre a origem da caça esportiva de aves desde o Século XIX e a situação dos efeitos ambientais do $\mathrm{Pb}$ como munição de caça, com foco em aves terrestres no sul do Brasil.

\section{MATERIAL E MÉTODOS}

Nesta revisão científica, utilizaram-se como principais fontes as publicações como artigos, revisões, notas científicas, livros digitalizados na internet, e sites de revistas científicas especializadas. O setor de periódicos impressos da Biblioteca UNISINOS, em São Leopoldo, RS foi pesquisado exaustivamente na busca de assuntos relacionados ao tema. Profissionais do setor de Ornitologia e Manejo de Fauna do Museu de Ciências Naturais (MCN) da Fundação Zoobotânica do Rio Grande do Sul (FZB-RS) foram entrevistados, bem como a biblioteca da instituição foi consultada na busca de trabalhos relacionados. Trabalhos, livros e referências do grupo de pesquisa em ecotoxicologia de $\mathrm{Pb} \mathrm{em}$ aves do Programa de Pós-graduação em Biologia (Diversidade e Manejo de Vida Silvestre) da UNISINOS também foram utilizados. Adicionalmente, foram contatados autores do exterior para disponibilizarem seus artigos.

\section{A ORIGEM DA CAÇA ESPORTIVA NO SÉCULO XIX}

A caça esportiva se profissionalizou no Reino Unido, onde os anglo-saxões reuniam no final do século XIX bibliografia relativa às espécies da Ásia e Índia (Finn 1911) que eram de interesse para a caça terrestre de seu país (Carnegie 1884), inclusive com caracterização ecológica e taxonômica pormenorizada de mais de uma centena de espécies. Nesta mesma época, nos E.U.A. a caça esportiva era praticada em 200 parques privados e clubes de caça (Huntington 1903). Estes ambientes de caça localizavam-se em áreas úmidas e terras altas [as espécies de aves cinegéticas de terras altas eram os galináceos (Jacus, Mutuns, Tetrazes e Faisões), columbídeos (Pombas) e, por vezes, ralídeos (Saracuras, Sanãs, Frangos d'água e Carquejas), 
charadrídeos (Batuíras) e scolopacídeos (Narcejas e Maçaricos)], manejadas com a finalidade de caça, da mesma forma que na Inglaterra. Os clubes organizavam as caçadas, regiam os procedimentos de participação, asseguravam e davam condições para que os locais de caça estivessem protegidos e adequados para a atividade (ex. suprimentos de grãos para fasianídeos, montagem de estandes de tiro e acessos às áreas úmidas), inclusive empregando guardas de caça (vigilantes que resguardavam os locais onde a caça era exercida). Naquela época, os E.U.A. já possuíam boa literatura disponível sobre vida selvagem e caça esportiva, onde instituições científicas e editoras independentes publicavam farta literatura sobre aves silvestres. Nesta época, também já se verificavam desequilíbrios populacionais resultantes da caça esportiva nos E.U.A., informações que chegavam aos caçadores por intermédio de publicações (Leffingwell 1888, Ogilvie-Grant 1896, 1897, Huntington 1903, Forbush 1912, 1913, 1917, Blanchan 1922). Adicionalmente, os norte-americanos pesquisavam sobre as aves de caça de outros países. Entre 1910 e 1920, Osgood \& Conover (1922) realizaram coletas juntamente com um inventário destas aves na Venezuela. Dentre elas, a descrição taxonômica, distribuição e comportamento de alguns tinamídeos florestais, ralídeos, galináceos, columbídeos e cracídeos. Posteriormente, Bump \& Bump (1969) pesquisaram exaustivamente a codorna-amarela [Nothura maculosa (Temminck, 1815), Tinamiformes, Tinamidae] na Argentina, com o intuito de avaliar a possibilidade de introdução desta espécie nos E.U.A., visto o seu bom valor para a caça.

Em 1937, o presidente dos E.U.A. Franklin D. Roosevelt assinou a Lei Pittman-Robertson que regulamentou a caça esportiva no país, instituindo taxas aos usuários como fundo de manejo e conservação de espécies e ecossistemas (US-FWS 2010). Legislações similares também passaram a existir no Canadá, México e Austrália. Segundo um informe da "National Survey of Fishing and Hunting in the Unites States", em Janeiro de 1961 havia 30 milhões de aficionados pela pesca e caça desportiva nos EUA e, entre 1955 e 1961 houve um aumento de $18 \%$ destes praticantes (Daciuk 1967).

\section{A CAÇA ESPORTIVA NO SUL DA AMÉRICA DO SUL}

$\mathrm{Na}$ década de 60 , a pesca e a caça eram as atividades recreacionais que reuniam mais adeptos na Argentina e no resto do mundo (Daciuk 1967). Naquele país, a caça foi regulamentada com a Lei Federal $n^{\circ}$ 13.908. Essa legislação caracterizava quatro formas de caça: caça indolente, desportiva, comercial e com fins científicos. A caça de aves terrestres era focada nos tinamídeos, sendo que dados de caça destes animais já eram registrados desde o início do Século XIX, na região do Chaco Argentino e Província de Buenos Aires (Arribáizaga 1918 e Série 1921 apud Menegheti 1982). Em determinadas épocas, a maioria dos abates eram com finalidades comerciais, inclusive com exportações para os E.U.A. e Reino Unido (Menegheti 1982).

No final do século XIX, a atividade de caça no estado de São Paulo (Brasil) começou a ser regulamentada, com normas para períodos e espécies campestres a serem abatidas e protegidas (Inhering 1902 apud Menegheti 1982, Menegheti 1985). No nível federal, a regulamentação da atividade efetivou-se pelo Código de Caça e Pesca promulgado em 1934 (Decreto Federal 23.672 de 02/01/1934). Contudo, o estado do Rio Grande do Sul com suas vastas extensões de campos, banhados, lagoas, açudes e lavouras de arroz foi o principal pólo cinegético do país até 2005 . $\mathrm{Na}$ década de 40 e 50, Burger-Marques \& Menegheti (1982) mencionam detalhes sobre as regulamentações utilizadas para a caça no estado a respeito das espécies, número de exemplares a serem abatidos, períodos, bem como os municípios onde a caça era permitida. Os caçadores eram cadastrados e legalizados, porém o número diminuiu com o passar dos anos (Tabela 1).

O estado do Rio Grande do Sul sobressaiu-se com uma rígida e controlada legislação, baseada em pesquisas e monitoramentos ornitológicos efetivos e contínuos, que indicavam as espécies de aves, épocas e locais de caça esportiva permitidos (Duarte 2005, Duarte et al. 2007). Nos últimos anos até 2005, as espécies legalizadas para caça eram anatídeos [marrecão, Netta peposaca (Vieillot, 1816); irerê, Dendrocygna viduata (Linnaeus, 1766) e marreca- 
caneleira, D. bicolor (Vieillot, 1816)], tinamídeos (codorna-amarela, N. maculosa) e columbídeos [asabranca, Patagioenas picazuro (Temminck, 1813) e avoante, Zenaida auriculata (Des Murs, 1847)]. Para N. maculosa, os estudos realizados nas microrregiões da Campanha Central/Ocidental e setor sul da Depressão Central do estado apontavam um período de dois anos e nove meses de moratória para que os estoques fossem preservados localmente (Duarte 2005). Em 2005, a Ação Civil Pública $n^{\circ}$. 2004.71.00.021481-2/RS proibiu a caça esportiva no Rio Grande do Sul, com a alegação de contaminação ambiental por $\mathrm{Pb}$, decorrente da munição de caça. Em países da Europa, ações semelhantes já foram efetivadas com a proibição total do metal ou parcial, em áreas úmidas.

Tabela 1. Ano de referência e número de caçadores legalizados no estado do Rio Grande do Sul, Brasil (Fonte: Menegheti 2005, Guadagnin et al. 2007)

Table 1. Year of reference and number of legalized hunters in the Rio Grande do Sul State, Brazil (Source: Menegheti 2005, Guadagnin et al. 2007).

\begin{tabular}{lc}
\hline Ano & Caçadores legalizados \\
\hline 1966 & 40.000 \\
1970 & 15.000 \\
1981 & 10.000 \\
2005 & 1.200 \\
\hline
\end{tabular}

\section{FONTES DE CHUMBO E SEUS EFEITOS AMBIENTAIS}

A munição de caça esportiva utilizada no Brasil e em outros países é o $\mathrm{Pb}$, metal que não demonstra função biológica em organismos vivos e resulta em efeitos adversos em diversos sistemas corporais de vertebrados (LSC 1987, Scheuhammer et al. 1998, US-DHHS 2007). Este metal está naturalmente presente no ambiente, assim como outros com características tóxicas conhecidas a organismos vivos (Mercúrio, Vanádio, Arsênico, Selênio e Cádmio) (White et al. 1980, Legat \& Brito 2010). Em 1987, o $\mathrm{Pb}$ foi o quinto metal mais usado no mundo, sendo o aditivo da gasolina a principal fonte de sua utilização (LSC 1987). Os usos de $\mathrm{Pb}$ na queima de combustíveis fósseis, agricultura, bem como na área industrial e doméstica contribuem para a incidência de altos níveis do poluente em determinadas áreas (White et al. 1980). Os centros urbanos são os principais pontos de emissão de $\mathrm{Pb}$ (US-DHHS 2007), com ênfase para o entorno de áreas de mineração e fundições de metais, contaminadas com efluentes industriais e com forte presença de veículos automotores (White et al. 1980, DeMent et al. 1987, LSC 1987, Franson 1996, Beyer et al. 2004, Malik \& Zeb 2009).

No último século, a mineração extensiva e o uso industrial resultaram no incremento da emissão do $\mathrm{Pb}$ na atmosfera que pode ser inalado por humanos e animais ou depositado no solo, corpos hídricos e vegetação (LSC 1987, Beyer et al. 2004). A toxicidade para humanos tem como vias principais a inalação e ingestão. Dependendo de inúmeros fatores, incluindo idade, sexo e estado nutricional, uma porcentagem de $\mathrm{Pb}$ ingerido ou inalado é eliminado, enquanto o restante pode ser absorvido ou incorporado (US-DHHS 2007). Em um homem adulto, de $10 \%$ a $15 \%$ do $\mathrm{Pb}$ inalado é absorvido pelo trato gastrointestinal, sendo o restante excretado (LSC 1987).

Os efeitos de absorção corporal de $\mathrm{Pb}$ em seres vivos pode resultar na doença chamada saturnismo. Em humanos os sintomas iniciais são sutis e inespecíficos e envolvem o sistema nervoso (fadiga, irritabilidade, distúrbios do sono, cefaleia, dificuldades de concentração, redução da libido), gastrointestinal (cólicas abdominais inespecíficas de fraca intensidade, anorexia, náusea, constipação intestinal, diarreia) e dor em membros inferiores (Brasil 2006). Em aves intoxicadas os sintomas são de um proventrículo distendido, fezes aquosas verdes, perda de peso, anemia e inclinação de postura, além de uma gama de mudanças sub-letais bioquímicas, fisiológicas e comportamentais (Pain et al. 2009). O diagnóstico da doença é realizado quando vários dados estão suficientemente disponíveis e consistentes para avaliação, tais como observações de campo, evidências físicas e funcionais objetivas da doença, juntamente com resultados laboratoriais (Franson 1996, Friend et al. 1999). O nível corporal de outros elementos também pode potencializar os efeitos tóxicos do $\mathrm{Pb}$, como por exemplo a ausência de Zinco ( $\mathrm{Zn}$ ), que protege a inibição da enzima Delta Aminolevulínico Desidratase 
(ALAD), essencial para a síntese de hemoglobina (US-DHHS 2007).

\section{A CONTAMINAÇÃo DE AVES POR Pb}

A caça esportiva de aves foi até pouco tempo predominantemente abastecida com munição a base de $\mathrm{Pb}$. Após o início da atividade, com o acréscimo acentuado de praticantes, este metal já indicava ser um poluente ambiental, sobretudo em corpos hídricos. A contaminação de aves aquáticas pelo $\mathrm{Pb}$ utilizado na munição de caça é reconhecida nos E.U.A. e Reino Unido desde 1874 (Grinnell 1894 apud Elder 1954). A primeira pesquisa sobre envenenamento dessas aves foi publicada em 1919 (Leopold 1986). Nesses locais, a concentração de $\mathrm{Pb}$ é mais acentuada no sedimento. $\mathrm{O} \mathrm{Pb}$ é ingerido juntamente com o forrageamento de anatídeos mergulhadores ("diving ducks") (Pace et al. 1999, Friend et al. 1999, Bennett et al. 2007). Em 1991, a caça com esta munição em corpos hídricos foi banida nos E.U.A. e, em 1999, também banida na caça de aves migratórias abatidas próximas de áreas úmidas no Canadá (Scheuhammer et al. 1998, Beintema 2003, Lenten 2005, Tranel \& Kimmel 2008, Pain et al. 2009). Recentemente na Argentina, a contaminação de $\mathrm{Pb}$ também foi confirmada para aves aquáticas (Ferreyra et al. 2009).

A contaminação de aves em geral por $\mathrm{Pb}$ pode ser o resultado do i) consumo direto de munição de $\mathrm{Pb}$ deflagrada (projéteis em esferas de $\mathrm{Pb}$ ou "chumbinhos"), pela ação da geofagia de aves (ingestão de minerais para auxílio da digestão mecânica da moela) (Best \& Gionfriddo 1991a, Connor et al. 1994, Gionfriddo \& Best 1996, Fischer et al. 2006, Fredricks et al. 2009) ou itens alimentares contaminados (Beyer et al. 2004, Scheuhammer 2009); ii) da ingestão de fragmentos de pesos para pesca ("chumbada"); ou iii) da exposição a tintas com base de $\mathrm{Pb}$ (Sileo \& Fefer 1987).

Em aves terrestres a contaminação era conhecida desde o final do século XIX (Calvert 1874 apud Rattner 2009), porém foi menos registrada $\mathrm{e}$ estudada em relação às aves aquáticas (Scheuhammer 2009, Pain et al. 2009). De qualquer forma, há registros de contaminação para mais de 59 espécies de aves terrestres (Fischer et al. 2006). A contaminação secundária de aves terrestres por $\mathrm{Pb}$ ocorre quando aves de rapina e/ou necrófagas ingerem presas, carcaças ou animais feridos e/ou debilitados contaminados (Benson et al. 1974, Carrington \& Mirarchi 1989, Fischer et al. 2006, Green et al. 2008, Nam \& Lee 2009).

Os dados apresentados de Franson (1996) demonstram os níveis de contaminação por $\mathrm{Pb}$ em aves terrestres das Ordens Falconiformes, Columbiformes e Galliformes; para esta última, o autor caracteriza o nível de contaminação através de resíduos de $\mathrm{Pb}$ no sangue, fígado e rim, de acordo com a severidade dos efeitos. Assim para a contaminação hepática por $\mathrm{Pb}$ os efeitos fisiológicos, como o ALAD, evidenciam-se com a presença de 2 a $6 \mathrm{mg} / \mathrm{kg}$ (peso úmido); sinais clínicos como anemia, lesões microscópicas dos tecidos, perda de peso, descoordenação muscular, diarreia e anorexia com níveis de $\mathrm{Pb}$ acima de $6 \mathrm{mg} / \mathrm{kg}$ e óbito com níveis de $\mathrm{Pb}$ acima de $15 \mathrm{mg} / \mathrm{kg}$.

Em aves aquáticas, os níveis de contaminação e efeitos são semelhantes. Pain (1996) categorizou que o comprometimento das funções biológicas e sinais externos de contaminação aparecem com níveis de $\mathrm{Pb}$ entre 6 e $15 \mathrm{mg} / \mathrm{kg}$ por peso úmido (ou aproximadamente 20 e $50 \mathrm{mg} / \mathrm{kg}$ por peso seco). Beyer et al. (2004) verificaram que em aves terrestres das espécies Colinus virginianus (Linnaeus, 1758) (Galliformes, Odonthophoridae) e Zenaida macroura (Linnaeus, 17858) (Columbiformes, Columbidae), os níveis de $\mathrm{Pb}$ no fígado ocorreram em concentrações que variavam de 0,16 e $1,2 \mathrm{mg} / \mathrm{kg}$ (peso seco), respectivamente, em áreas contaminadas próximas à mineração e fundições; o autor conclui que estas espécies haviam se contaminado com sedimentos e não com munição de caça.

No Rio Grande do Sul, os níveis do $\mathrm{Pb}$ em aves campestres (N. maculosa) e no solo foram analisados por Lau (2011) em setores com histórico de caça e com ausência de outras fontes de $\mathrm{Pb}$. $\mathrm{O}$ autor não encontrou evidências de ingestão de munição de $\mathrm{Pb}$ por geofagia e, os níveis hepáticos do metal estavam abaixo do limiar considerado tóxico (inferiores a 1mg/ $\mathrm{kg}$ ). Juntamente analisadas, as amostras de solo não apresentaram munição deflagrada e contaminação de $\mathrm{Pb}$, e demonstraram níveis abaixo do limiar considerado tóxico (inferiores a $100 \mathrm{mg} / \mathrm{kg}$ ). 


\section{CONSIDERAÇÕES FINAIS}

O emprego de $\mathrm{Pb}$ como munição de caça causa contaminação direta e indireta da vida silvestre no ambiente aquático e, em menor escala, no ambiente terrestre. Em outros países, a proibição de munição a base de $\mathrm{Pb}$ somente em áreas úmidas é ineficaz, visto o não atendimento da conduta por alguns caçadores e o direcionamento desta caça para áreas terrestres, contaminando aves deste ambiente. No Brasil, principalmente no estado do Rio Grande do Sul onde a caça campestre é histórica e foi controlada por órgãos competentes por muitos anos, não se buscou a iniciativa de inserir munição de caça alternativa ao $\mathrm{Pb}$ e sim foi efetivada o fim da atividade. No entanto, a caça ilegal ainda é uma atividade comum principalmente na região campestre do estado e, dessa maneira, a contaminação por $\mathrm{Pb}$ ainda é potencialmente uma ameaça importante às aves e outras espécies em ambientes silvestres e rurais.

Os estudos ecotoxicológicos com $\mathrm{Pb}$ em aves no Brasil ainda são escassos e, embora $\mathrm{o} \mathrm{Pb}$ oriundo de munições possa ser uma fonte importante de contaminação, a sua contribuição relativa para a contaminação do ambiente e vida silvestre ainda é pouco estudado. Dessa maneira, os estudos futuros podem focar na origem/fonte (antrópica e/ou natural) do $\mathrm{Pb}$ através de estudos de seus isótopos.

\section{AGRADECIMENTOS}

Agradecimentos ao Dr. Demetrio Luis Guadgnin, Dr. Luis Fernando Carvalho Perello, Dr. Leonardo Maltchik Garcia, Dra. Cristina Stenert, Dra. Maria Virgínia Petry e Fundação Zoobotânica do Rio Grande do Sul.

\section{REFERÊNCIAS}

Beintema, N. 2003. Non-toxic shot: a path towards sustainable use of waterbird resource. Technical Series No. 3; p. 32. Bonn: UNEP/AEWA Secretariat. Disponível em http://www.unepaewa.org/en/publication/non-toxic-shot-path-towardssustainable-use-waterbird-resource-ts-no-3.

Bennett, J. R., Kaufmann, C. A., Koch, I., Sova, J., \& Reiner, K. J. 2007. Ecological risk assessment of lead contamination at rifle and pistol ranges using techniques to account for site characteristics. Science of the Total Environment, 1(1), 91101. DOI: $10.1016 /$ j.scitotenv.2006.12.040.

Benson, W. W., Pharaoh, B., \& Miller, P. 1974. Lead poisoning in a bird of prey. Bulletin of Environmental Contamination \& Toxicology, 11(2), 105-108. DOI: 10.1007/BF01684587.

Best, L. B., \& Gionfriddo, J. P. 1991a. Characterization of grit used by cornfield birds. Wilson Bulletin, 103(1), 68-82.

Beyer, W. N., Dalgarn, J., Dudding, S., French, J., Mateo, R., Miesner, J., Sileo, L., \& Spann, J. 2004. Zinc and lead poisoning in wild birds in tri-state mining district (Oklahoma, Kansas and Missouri). Archives of Environmental Contamination and Toxicology, 48(1), 108-117. DOI: 10.1007/s00244-004-0010-7.

Blanchan, N. (Ed.). 1922. Game birds: Life history of one hundred and seventy birds of prey, game birds and water-fowls. New York: Doubleday: p. 422.

Brasil. 1934. Decreto n ${ }^{\circ} 23.672$, de 2 de janeiro de 1934. Disponível em http://www2.camara.leg.br/legin/fed/decret/1930-1939/ decreto-23672-2-janeiro-1934-498613-publicacaooriginal-1pe.html.

Brasil. 2006. Ministério da Saúde. Secretaria de Atenção à Saúde. Departamento de Ações Programáticas Estratégicas. Atenção à saúde dos trabalhadores expostos ao chumbo metálico. Série A. Normas e Manuais Técnicos. Brasília, DF: Editora do Ministério da Saúde: p. 44.

Burger-marques, M. I., \& Menegheti, J. O. 1982. Portaria de caça: um instrumento para a conservação da fauna. Natureza em Revista, 9, 14-20.

Bump, G., \& Bump, J. W. 1969. A study of spotted tinamous and the pale spotted tinamous of Argentina. Relatório Técnico. United States Fish and Wildlife Service (USFWS), Virgínia. p. 160.

Carnegie, W. (Ed.). 1884. Pratical Game Preserving: containing the fullest directions for rearing and preserving both winged and Ground game, and destroying vermin. London: L. Upcott Gill: p. 598.

Carrington, M. E., \& Mirarchi, R. E. 1989. Effects of lead shot ingestion on free-ranging mourning doves. Bulletin of Environmental Contamination and Toxicology, 43(2), 173179. DOI: $10.1007 / \mathrm{BF} 01701744$.

Connor, E. E., Scanlon, P. F., \& Kirkpatrick, R. L. 1994. Bioavailability of lead from contaminated sediment in northern bobwhites, Colinus virginianus. Archives of Environmental Contamination and Toxicology, 27(1), 60-63. DOI: 10.1007/ BF00203889.

Costa, R. G. A. 2006. Caça e saturnismo em aves silvestres: uma questão ainda obscura na ornitologia brasileira. Atualidades Ornitológicas, 132, 18-19.

Daciuk, J. 1967. Consideración acerca de los fundamentos de la protección y conservación de la fauna nativa. In: Dirección de Parques Nacionales (Ed.), Anales de Parques Nacionales. pp. 43-96. Buenos Aires: Secretaria de Estado de Agricultura y Ganaderia de La Nación.

Davidson, W. R., \& Nettles, V. F. 1997. Field manual of wildlife diseases in the Southeastern United States. 2nd ed. Athens, GA: Southeastern Cooperative Wildlife Disease Study (SCWDS): p. 448.

DeMent, S. H., Chisolm, J. J. Jr., Eckhaus, M. A., \& Strandberg, J. D. 1987. Toxic lead exposure in the urban rock dove. Journal of Wildlife Diseases, 23(2), 273-278. DOI: 10.7589/00903558-23.2.273. 
Duarte, M. M. Programa de Pesquisa e Monitoramento de Fauna Cinegética do Rio Grande do Sul - Período 2004-2005. Relatório Técnico. Fundação Zoobotânica do Rio Grande do Sul (FZB-RS), Porto Alegre. p. 67.

Duarte, M. M., Bencke, G. A., \& Menegueti, J. O. 2007. Avaliação da abundância e tendências populacionais de Nothura maculosa (Perdiz) no Rio Grande do Sul. Programa de Pesquisa e Monitoramento de Fauna Cinegética do Rio Grande do Sul, Período de 2006-2007. Relatório Técnico. Fundação Zoobotânica do Rio Grande do Sul (FZB-RS), Porto Alegre. p. 104.

Ferreyra, H., Romano, M., \& Uhart, M. 2009. Recent and chronic exposure of wild ducks to lead in human-modified wetlands in Santa Fe Province, Argentina. Journal of Wildlife Diseases, 45(3), 823-827. DOI: 10.7589/0090-3558-45.3.823.

Finn, F. (Ed.). 1911. The game birds of India and Asia. Calcutta: Thacker, Spink \& Company: p. 212.

Fischer, I. J., Pain, D. J., \& Thomas, V. G. 2006. A review of lead poisoning from ammunition sources in terrestrial birds. Biological Conservation, 131(3), 421-432. DOI:10.1016/ j.biocon.2006.02.018.

Flores, E. M. M., \& Martins, A. F. 1997. Distribution of trace elements in egg samples collected near coal power plants. Journal of Environmental Quality, 26(3), 744-748. DOI: 10.2134/jeq1997.00472425002600030021x.

Forbush, E. H. (Ed.). 1912. A history of the game birds, wildfowl and shorebirds of Massachusetts and adjacent States. Massachusetts: Wright \& Potter Printing Company: p. 708.

Forbush, E. H. (Ed.). 1913. Game birds of America. New York: The Mentor Association Inc.: p. 30. DOI: 10.5962/ bhl.title.56669

Forbush, E. H. (Ed.). 1917. Important American game birds: their ranges, habits and the hunting. Delawere: E. I. Du Pont de Nemours \& Company: p. 60.

Franson, J. C. 1996. Interpretation of tissue lead residues in birds other than waterfowl. In: W. N. Beyer, G. H. Heinz \& A. W. Redmon-Norwood (Eds.), Environmental contaminants in wildlife: interpreting tissue concentrations. pp. 265-279. Boca Raton: Lewis Publishers.

Fredricks, T. B, Fedynich, A. M., Benn, S., \& Ford, L. 2009. Environmental contaminants in white-winged doves (Zenaida asiatica asiatica) from the Lower Rio Grande Valley of Texas, USA. Archives of Environmental Contamination and Toxicology, 57(2), 387-396. DOI: 10.1007/s00244-0089274-7.

Friend, M., Franson, J. C., \& Ciganovich, E. A. 1999. Field manual of wildlife diseases: general field procedures and diseases of birds. Information and Technology Report No. 1999-001; p. 438. Madison: Biological Resources Division.

Gionfriddo, J. P., \& Best, L. B. 1996. Grit-use patterns in North American birds: the influence of diet, body size, and gender. The Wilson Bulletin, 108(4), 685-696.

Green, R. E., Hunt, W. G., Parish, C. N., \& Newton, I. 2008. Effectiveness of action to reduce exposure of free-ranging California condors in Arizona and Utah to lead from spent ammunition. PLoS One, 3(12), e4022. DOI: 10.1371/ journal.pone.0004022
Elder, W. H. 1954. The effect of lead poisoning on the fertility and fecundity of domestic Mallard ducks. The Journal of Wildlife Management, 18(3), 315-323. DOI: 10.2307/ 3797018 .

Guadagnin, D. L., Perello, L. F. C., \& Menegheti, J. O. 2007. Current situation of leisure hunting and wetlands management in Rio Grande do Sul State, South Brazil. Neotropical Biology and Conservation, 2(2), 63-70.

Huntington, D. W. (Ed.). 1903. Our feathered game. A handbook of the north American game birds. New York: Charles Scribner's Sons: p. 478.

Lau, R. 2011. Contaminação por chumbo de munição de caça em aves e solos de campos do Bioma Pampa, Brasil. Laboratório de Ecologia e Conservação de Ecossistemas Aquáticos (LECEA) da Universidade do Vale do Rio dos Sinos. p. 58.

Leffingwell, W. B. (Ed.). 1888. Wild fowl shooting. Containing scientific and practical descriptions of wild fowl: their resorts, habits, flights and the successful method of hunting them. Chicago: Rand, McNally \& Company: p. 418.

Legat, L. N. A., \& Brito, J. L. 2010. O mercúrio em cetáceos (Mammalia, Cetacea): uma revisão. Oecologia Australis, 14(4), 1021-1035. DOI: $10.4257 /$ oeco.2010.1404.12.

Lenten, B. 2005. The role of the AEWA in phasing out lead shot for hunting in wetlands. Bird Conservation International, 15(2), 161-163. DOI: 10.1017/S0959270905000122.

Leopold, A. (Ed.). 1986. Game management. Madison: University of Wisconsin Press: p. 481.

LSC (Lead in Soil Committee). 1987. Review and recommendations on a lead in soil guideline. Relatório Técnico. Ministry of the Environment, Ontario. p. 122.

Malik, R. N., \& Zeb, N. 2009. Assessment of environmental contamination using feathers of Bubulcus ibis L., as a biomonitor of heavy metal pollution, Pakistan. Ecotoxicology, 18(5), 522-536. DOI: 10.1007/s10646-009-0310-9.

Menegheti, J. O. 1982. Curiosidades sobre as atividades da caça à perdiz. Natureza em Revista (Brazil), 9, 28-30.

Menegheti, J. O. 1985. Características da caça e seus efeitos sobre a população de Nothura maculosa (Temminck, 1815) no Rio Grande do Sul. Iheringia, série Miscelânea, 1, 87-100.

Menegheti, J. O. 2005. Avaliação dos efeitos da estiagem que assolou o Rio Grande do Sul durante o verão de 2005 sobre as populações locais de perdiz. Relatório Técnico. Museu de Ciências Naturais, Fundação Zoobotânica do Rio Grande do Sul, Rio Grande do Sul. p. 12.

Nam, D. H., \& Lee, D. P. 2009. Abnormal lead exposure in globally threatened Cinereous vultures (Aegypius monachus) wintering in South Korea. Ecotoxicology, 18(2), 225-229. DOI: 10.1007/ s10646-008-0275-0.

Ogilvie-Grant, W. R. (Ed.). 1896. A hand-book to the game-birds Vol. I. London: Edward Lloyd, Limited: p. 376.

Ogilvie-Grant, W. R. 1897. A hand-book to the game-birds Vol. II. London: Edward Lloyd, Limited: p. 397.

Osgood, W. H., \& Conover, H. B. (Eds.). 1922. Game birds from northwestern Venezuela. Chicago: Field Museum of Natural History. Zoological Series Vol. XII, No. 3: p. 38. DOI: 10.5962/ bhl.title.5580.

Pace, R. M. R., Hohman, W. L., \& Custer, T. W. 1999. Lead 
effects on body and organ size of wintering canvasbacks Aythya valisineria in Louisiana. Wildlife Biology, 5(1), 3-10.

Pain, D. J. 1996. Lead in waterfowl. In: W. N. Beyer, G. H. Heinz \& A. W. Redmon-Norwood (Eds.), Environmental contaminants in Wildlife: interpreting tissue concentrations. pp. 51-264. Boca Raton: CRC Press.

Pain, D. J., Fisher, I. J., \& Thomas, V. G. 2009. A global update of lead poisoning in terrestrial birds from ammunition sources. In: R. T. Watson, M. Fuller, M. Pokras \& W. G. Hunt (Eds.), Ingestion of lead from spent ammunition: implications for wildlife and humans. pp. 99-118. Idaho: The Peregrine Fund. DOI: $10.4080 /$ ilsa.2009.0108.

Rattner, A. B. 2009. History of wildlife toxicology. Ecotoxicology, 18(7), 773-783. DOI: 10.1007/s10646-009-0354-x.

Scheuhammer, A. M., Perrault, J. A., Routhier, E., Braune, B. M., \& Campbell, G. D. 1998. Elevated lead concentrations in edible portions of game birds harvested with lead shot. Environmental Pollution, 102(2-3), 251-257. DOI: 10.1016/S02697491(98)00083-9.

Scheuhammer, A. M. 2009. Historical perspective on the hazards of environmental lead from ammunition and fishing weights in Canada. In: R. T. Watson, M. Fuller, M. Pokras \& W. G. Hunt (Eds.), Ingestion of lead from spent ammunition: implications for wildlife and humans. pp. 61-67. Idaho: The Peregrine Fund. DOI: 10.4080/ilsa.2009.0108.

Sileo, L., \& Fefer, S. L. 1987. Paint chip poisoning in Laysan albatross at Midway Atoll. Journal of Wildlife Diseases, 23(3), 432-437. DOI: 10.7589/0090-3558-23.3.432.

Tranel, M. A., \& Kimmel, R. O. 2008. Nontoxic and lead shot literature review. Relatório Técnico. Minnesota Department of Natural Resources (Minnesota DNR), Section of Wildlife, Minnesota. p. 40.

Tsutiya, M. T. 1999. Metais pesados: o principal fator limitante para o uso agrícola de biossólidos das estações de tratamento de esgotos. In: $20^{\circ}$ Congresso Brasileiro de Engenharia Sanitária e Ambiental, Rio de Janeiro, RJ, 753-761. Disponível em http://www.bvsde.paho.org/bvsaidis/brasil20/i-140.pdf.

US-DHHS (U.S. Department of Health and Human Services). 2007. Toxicological profile for lead. Relatório Técnico. Agency for Toxic Substances and Disease Registry, Atlanta. p. 582.

US-FWS (US. Fish \& Wildlife Service). 2010. Federal Aid Division - The Pittman-Robertson federal aid in wildlife restoration act. Disponível em http://www.fws.gov/southeast/federalaid/ pittmanrobertson.html.

US-FWS (US. Fish \& Wildlife Service). 2014. Nontoxic shot regulations for hunting waterfowl and coots in the U.S. Disponível em https://www.fws.gov/birds/bird-enthusiasts/ hunting/nontoxic.php.

White, D. H., King, K. A., \& Prouty, R. M. 1980. Significance of Organochlorine and heavy metal residues in wintering shorebirds at Corpus Christi, Texas, 1976-1977. Pesticides Monitoring Journal, 14(2), 58-63. 